\title{
Coulomb explosion of deuterium cationic clusters
}

\author{
I. A. Howard, ${ }^{1}$ J. A. Alonso, ${ }^{2,3}$ N. H. March, ${ }^{1,4}$ A. Rubio, ${ }^{2,5}$ and C. Van Alsenoy ${ }^{6}$ \\ ${ }^{1}$ Department of Physics, University of Antwerp, Groenenborgerlaan 171, B-2020 Antwerp, Belgium \\ ${ }^{2}$ Donostia International Physics Center (DIPC), E-20018 San Sebastián, Spain \\ ${ }^{3}$ Departmento de Física Teorica, Universidad de Valladolid, 47001 Valladolid, Spain \\ ${ }^{4}$ Oxford University, Oxford, England \\ ${ }^{5}$ Departamento de Física de Materiales, Universidad del País Vasco, and Centro Mixto CSIC/UPV, Paseo Manuel Lardizabal 3, \\ E-20018 San Sebastián, Spain \\ ${ }^{6}$ Department of Chemistry, University of Antwerp, Universiteitsplein 1, B-2610 Antwerp, Belgium
}

(Received 11 August 2003; published 16 December 2003)

\begin{abstract}
The recent experiments of Zweiback et al. on deuterium clusters irradiated with intense laser beams yielded D-D fusion. This has motivated us to carry out quantum-chemical calculations on a number of deuterium clusters, both neutral and cationic. For doubly charged clusters, in particular $\mathrm{D}_{31}{ }^{2+}$, and plainly for more highly charged assemblies, Coulomb explosion of the cluster is in evidence. The quantum-chemical results we present strongly suggest that fragmentation occurs with small groups of deuterium atoms breaking away from the main cluster, such as to leave both small and large fragments with a net charge not exceeding $+|e|$.
\end{abstract}

DOI: 10.1103/PhysRevA.68.065201

PACS number(s): $36.40 .-\mathrm{c}$

\section{INTRODUCTION}

Hydrogen clusters are a typical example of molecular clusters, responding to the formula $\left(\mathrm{H}_{2}\right)_{N}$. The binding energy of the two hydrogen atoms in a molecule is high, $4.8 \mathrm{eV}$. On the other hand, the binding between the different molecules in the cluster is very weak. Detecting and analyzing cluster sizes in a typical mass spectrometric experiment requires the ionization of the clusters [1]. Ionization of the clusters is a local process affecting a molecule, which is usually followed by the reaction with another molecule of the cluster; in our case

$$
\mathrm{H}_{2}^{+}+\mathrm{H}_{2} \rightarrow \mathrm{H}_{3}^{+}+\mathrm{H}
$$

leading to a charged trimer $\mathrm{H}_{3}{ }^{+}$. The neutral $\mathrm{H}$ atom is ejected (and perhaps some neutral $\mathrm{H}_{2}$ molecules also) leaving an odd number of atoms in a positively charged cluster, finally arriving at the formula $\mathrm{H}_{3}{ }^{+}\left(\mathrm{H}_{2}\right)_{n}$, characteristic of the singly charged clusters [2]. The structure of those clusters, mainly for small $n$, has been studied by densityfunctional [3-5] and quantum-chemical methods [6-9]. The picture coming from those works is that the $\mathrm{H}_{2}$ molecules form shells around the $\mathrm{H}_{3}{ }^{+}$core (like solvation shells). The first shell is formed in $\mathrm{H}_{3}{ }^{+}\left(\mathrm{H}_{2}\right)_{3}$. In this case, the three $\mathrm{H}_{2}$ molecules surround the $\mathrm{H}_{3}{ }^{+}$triangular core, and each molecule is close to one of the $\mathrm{H}$ atoms. The center of mass of each $\mathrm{H}_{2}$ molecule is on the plane formed by the nuclei of the triangular core and the molecular axis is perpendicular to that plane. In the second shell the molecules are, evidently, at longer distances from the triangular core. In a recent experiment Gobet et al. studied the collisions of $\mathrm{H}_{3}{ }^{+}\left(\mathrm{H}_{2}\right)_{n}$ clusters and $\mathrm{He}$ atoms [2]. From the analysis of the fragmentation of size-selected clusters Gobet et al. were able to construct a caloric curve (excitation energy versus temperature) for the microcanonical cluster ion ensemble. This caloric curve exhibits the form of a typical first-order phase transition in a finite system, which was identified as a transition from a bound cluster to the gas phase.

Another interesting experiment involves large deuterium clusters, with radii of $25 \AA$ or more [10]. Irradiating a molecular beam of those large clusters with an intense pulsed femtosecond laser produces a very large ionization of the clusters, which lose most of their electrons. A Coulomb explosion of the nuclear skeleton then occurs, and the collisions between nuclei flying out from different clusters (with kinetic energies of several tens of $\mathrm{keV}$ ) yield D-D fusion, as observed by the emitted neutrons.

Motivated by those experiments we have studied the stability of medium-sized clusters, in particular $\mathrm{D}_{31}{ }^{+}$, against additional ionization. We find that the clusters fragment due to the strong Coulombic repulsion between the unbalanced charges. The fragmentation is very asymmetrical and it is characterized by the emission of $\mathrm{D}_{2}^{+}$dimers.

\section{QUANTUM CHEMICAL CALCULATIONS ON NEUTRAL AND CATIONIC DEUTERIUM CLUSTERS: GEOMETRY, ELECTRONIC STRUCTURE, AND COULOMB EXPLOSION}

In our preliminary work on small cationic clusters of hydrogen isotopes [9], one of the clusters studied was $\mathrm{D}_{13}{ }^{+}$. This was treated at Hartree-Fock (HF) plus MP2 (secondorder Möller-Plesset) level to find the optimized geometry and the corresponding ground-state electronic structure. In the present paper we have refined our study of this cluster by working to MP4(SDQ) level, i.e., fourth-order Möller-Plesset using the space of single, double, and quadruple substitutions. The calculations reported here were carried out using the GAUSSIAN 98 [11] program package, with the 6-31G** basis. The normal-mode frequencies are recorded in Table I at the three levels of approximation indicated HF, MP2, and MP4. To calculational accuracy, the frequencies scale as $M^{-1 / 2}$, where $M$ is the isotopic mass (that is, the frequencies tabulated for $\mathrm{D}_{13}{ }^{+}$could be modified to yield those for hy- 
TABLE I. Calculated normal mode frequencies (in $\mathrm{cm}^{-1}$ ) for $\mathrm{D}_{13}{ }^{+}$at HF, MP2, and MP4 levels.

\begin{tabular}{|c|c|c|}
\hline \multicolumn{3}{|c|}{$\mathrm{D}_{13}{ }^{1+}$ normal mode frequencies $\left(\mathrm{cm}^{-1}\right)$} \\
\hline HF & MP2 & MP4 \\
\hline 10. & 12. & 15. \\
\hline 39. & 39. & 39. \\
\hline 49. & 53. & 53. \\
\hline 62. & 60. & 59. \\
\hline 64. & 62. & 60. \\
\hline 70. & 71. & 69. \\
\hline 71. & 76. & 73. \\
\hline 73. & 81. & 80. \\
\hline 84. & 83. & 85. \\
\hline 92. & 86. & 87. \\
\hline 94. & 92. & 98. \\
\hline 100. & 109. & 108. \\
\hline 104. & 111. & 115. \\
\hline 111. & 131. & 127. \\
\hline 149. & 132. & 134. \\
\hline 162. & 145. & 144. \\
\hline 203. & 223. & 220. \\
\hline 224. & 235. & 233. \\
\hline 236. & 243. & 245. \\
\hline 357. & 387. & 379. \\
\hline 360. & 388. & 385. \\
\hline 392. & 413. & 408. \\
\hline 433. & 533. & 530. \\
\hline 469. & 547. & 544. \\
\hline 470. & 550. & 552. \\
\hline 1975. & 1885. & 1867. \\
\hline 1990. & \begin{tabular}{|l}
1904. \\
\end{tabular} & 1885. \\
\hline 2520 . & 2463. & 2454. \\
\hline 3195. & 3168. & 3123. \\
\hline 3196. & 3170. & 3129. \\
\hline 3199. & 3173. & 3131. \\
\hline 3256. & 3237. & 3189. \\
\hline 3257. & 3238. & 3191. \\
\hline
\end{tabular}

drogen or for tritium by simple $M^{-1 / 2}$ scaling). Later, further refinements may prove necessary, but the main points to be emphasized from the table are that (i) all the frequencies are real, i.e., the geometrical structure at the HF, MP2, and MP4 levels is stable relative to small nuclear displacements and (ii) there is fair convergence in adding MP corrections. The five highest frequencies, corresponding to $\mathrm{D}_{2}$ intramolecular vibrations, decrease by an average of $0.73 \%$ in going from HF to MP2 level, and by a further $1.40 \%$ between MP2 and MP4. Correspondingly, the three next highest frequencies, which are essentially intramolecular modes of the trimer, decrease by an average of $3.71 \%$ in going from HF to MP2, and by $0.77 \%$ from MP2 to MP4. We may also compare binding energies of the cluster, relative to an isolated charged trimer and five isolated neutral dimers, at the three different levels of approximation; then we find that at HF level the cluster is bound by $0.42 \mathrm{eV}$, while at both MP2 and MP4 levels it is bound by $0.53 \mathrm{eV}$.

We show in Fig. 1 the refinements, especially in interatomic distances, in the $\mathrm{D}_{13}{ }^{+}$structure due to the addition of two levels of MP corrections to the zeroth-order HartreeFock treatment. The structure of the cluster is essentially the same at the three levels, HF, MP2, and MP4, although the interatomic distances change slightly. Within the equilateral

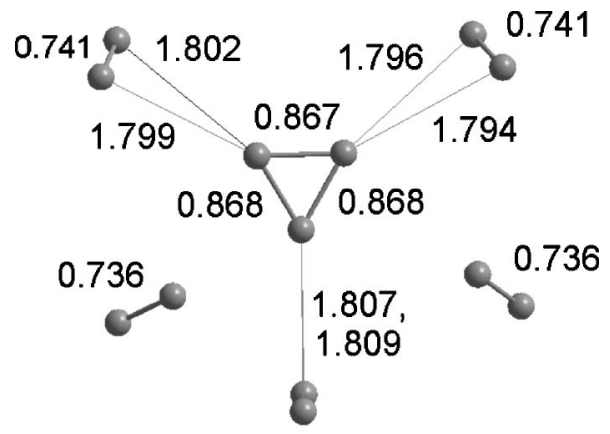

(a)

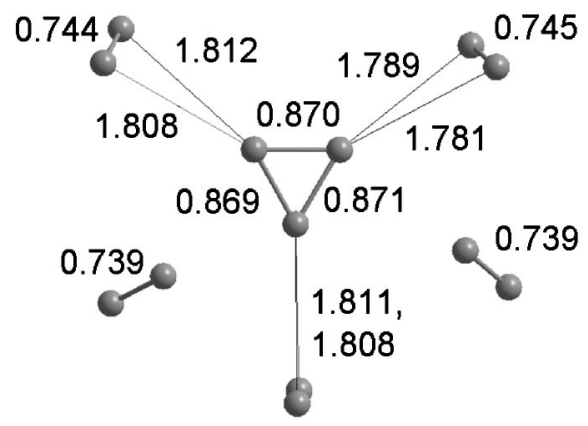

(b)

FIG. 1. Shows especially changes in intermolecular D-D distances on refinement of quantum-chemical calculations from (a) the MP2 to (b) the MP4 level for the cluster $\mathrm{D}_{13}{ }^{+}$. All distances are in angstroms.

singly charged trimer core, $\mathrm{D}_{3}{ }^{+}$, the average interatomic distance varies from $0.861 \AA$ at HF level to $0.868 \AA$ at MP2 and $0.870 \AA$ at MP4 level. This $\mathrm{D}_{3}{ }^{+}$core is surrounded by the first "shell" of $\mathrm{D}_{2}$ molecules; the distances from the D atoms in this shell to the nearest D atom in the trimer core range from an average of $1.931 \AA$ at HF level to $1.801 \AA$ at MP2 and $1.802 \AA$ at MP4 levels. Then the remaining two $\mathrm{D}_{2}$ molecules are part of a second shell at a distance from the trimer core which decreases from an average of $2.953 \AA$ within HF to $2.868 \AA$ at MP2 and to $2.855 \AA$ at MP4 level.

Having considered a small, singly charged cationic cluster of deuterium atoms, we turn to the Coulomb explosion of multiply charged deuterium clusters. We have considered a number of clusters and have chosen first a large doubly charged cluster, $\mathrm{D}_{31}{ }^{2+}$, to illustrate the main results. As the initial state we took the geometry of the singly charged $\mathrm{D}_{31}{ }^{+}$ cluster optimized at the Hartree-Fock level. The net charge of the cluster was then increased to $+2|e|$ by removing one electron from the highest occupied molecular orbital and the cluster was allowed to relax. A geometry optimization, using the Berny optimization algorithm, was carried out at the Hartree-Fock level (the results presented above for the singly charged cluster indicate that the use of MP2 or MP4 corrections will not change the picture). Figure 2, which shows the cluster geometry after 186 optimization cycles, makes it plain that the doubly charged cluster is unstable due to the large Coulomb repulsion between the two unbalanced posi- 


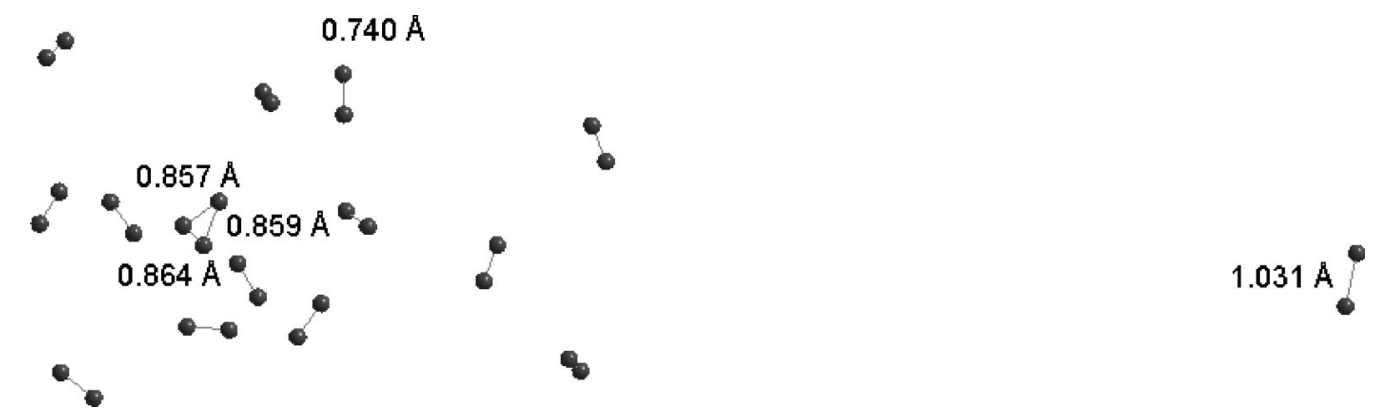

FIG. 2. Result of the Coulomb fragmentation of the doubly charged deuterium cluster $\mathrm{D}_{31}{ }^{2+}$. The picture shows a singly charged cationic dimer split off the large remaining singly charged fragment. The charge of the large fragment resides dominantly on the singly charged cationic trimer $\mathrm{D}_{3}{ }^{+}$, which is seen to be an almost equilateral triangle with bond length $\sim 0.86 \AA$.

tive charges. The cluster undergoes Coulomb fragmentation. A small fragment, the singly charged dimer $\mathrm{D}_{2}{ }^{+}$, has split off from the cluster. Its bond length has increased to $1.031 \AA$; this is about $30 \%$ larger than the bond length of the neutral molecules in the cluster. The large fragment contains the almost equilateral cationic trimer, with bond length $\sim 0.86 \AA$ and net charge $+0.82|e|$, obtained from a Mulliken population analysis. Both fragments are stable at the Hartree-Fock level. This result strongly suggests the preference for very unsymmetrical channels of fragmentation, similar to the case of Coulomb fragmentation in metallic clusters $[12,13]$.

A more dramatic case of Coulombic explosion is depicted in Fig. 3, where the parent cluster is $\mathrm{D}_{31}{ }^{4+}$. Again, the initial geometry was that of $\mathrm{D}_{31}{ }^{+}$, and the net charge was increased to $+4|e|$ by removing three electrons. After 100 optimization cycles, three singly charged dimers have split away from the central cluster. The large remaining fragment $\mathrm{D}_{25}{ }^{+}$is centered around the deuterium trimer, which carries a net charge of $+0.88|e|$. The large fragment is again stable at the Hartree-Fock level.

\section{SUMMARY AND FUTURE DIRECTIONS}

Our main findings in this Brief Report are based on what we can usefully view as a theoretical approach which recog-

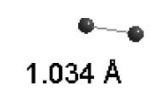

nizes in such deuterium clusters, neutral or charged, the existence of two expansion parameters. The first is the correlation energy $\epsilon$, which was defined by Löwdin as the difference of total energies,

$$
\epsilon=E_{\text {exact }}-E_{\text {Hartree }- \text { Fock }} .
$$

Essentially, the smallness of the ratio $\epsilon / E_{\text {exact }}$, especially for the larger clusters considered here, is the reason for the relative effectiveness of low-order Möller-Plesset perturbation theory, since the zeroth-order term of such an expansion is the Hartree-Fock solution. Returning to Table I, giving the vibrational frequencies of $\mathrm{D}_{13}{ }^{+}$, it is satisfying that for the majority the differences between MP2 and MP4 values are modest, though $\sim 40 \mathrm{~cm}^{-1}$ discrepancies appear at the highest frequencies. In the ground-state geometry, the intermolecular distances represent the only significant changes in going from the MP2 to MP4 level of accuracy.

As to Coulomb explosion, our calculations make it quite plain that the doubly charged cationic cluster $\mathrm{D}_{31}{ }^{2+}$ undergoes fragmentation, with a singly charged dimer clearly ejected from the remaining large fragment, which has a sin-
FIG. 3. Result of the Coulomb explosion of the cationic cluster $\mathrm{D}_{31}{ }^{4+}$. Three singly charged dimers are emitted, and the large remaining fragment is centered on a singly charged trimer. The very unsymmetrical character of the fragmentation brought about by Coulomb explosion is again in evidence.

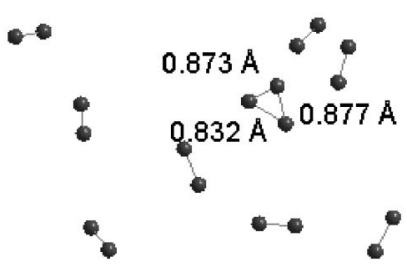


gly charged deuterium trimer at its core. When the cationic charge is increased to +4 , three singly charged dimers are ejected.

We return to the second expansion parameter relevant to such clusters of isotopes of hydrogen. This involves the ratio $m / M$, where $m$ is the electronic and $M$ the isotopic hydrogen mass. It is fair to say that this parameter, in our calculations to date, is treated less satisfactorily than the first parameter, namely, the correlation energy $\epsilon$. The immediate illustration of this, which has already been touched on, is that to the level of accuracy corresponding to the vibrational frequencies in Table I, the frequencies tabulated for the $\mathrm{D}_{13}{ }^{+}$cationic cluster could be modified to yield those for hydrogen or for tritium by simple $M^{-1 / 2}$ scaling. We expect future calculations to deal more accurately with this second expansion parameter $m / M$, but this would seem to involve substantial increase in computational effort, as is evident, for example, in the classic review of Öhrn et al. [14]

\section{ACKNOWLEDGMENTS}

I.A.H. and N.H.M. wish to acknowledge that their contribution to the present study was made during a visit to the Donostia International Physics Center (DIPC), and to thank Professor P. M. Echenique for generous hospitality. I.A.H. acknowledges support from the IWT-Flemish region. N.H.M. also acknowledges partial support from ONR for work on the many-electron theory of molecules and clusters. Dr. P. Schmidt of that Office is especially thanked for his support and motivation. The work of J.A.A. was supported by MCyT of Spain (Grant No. MAT2002-04499-C02-01), Junta de Castilla y León (Grant No.CO01/102), and by DIPC. A.R. thanks the EC research training network NANOPHASE (Grant No. HPRN-CT-2000-00167), the Spanish MCyT (Grant No. MAT2001-0946), and UPV/EHU (Grant No. 9/UPV 00206.215-13639/2001) for support.
[1] B. Farizon, M. Farizon, M. J. Gaillard, F. Gobet, M. Carré, J. P. Buchet, P. Scheier, and T. D. Märk, Phys. Rev. Lett. 81, 4108 (1998).

[2] F. Gobet, B. Farizon, M. Farizon, M. J. Gaillard, J. P. Buchet, M. Carré, P. Scheier, and T. D. Märk, Phys. Rev. Lett. 89, 183403 (2002); F. Gobet, B. Farizon, M. Farizon, M.J. Gaillard, J.P. Buchet, and M. Carré, ibid. 87, 203401 (2001).

[3] I. Stich, D. Marx, M. Parinello, and K. Terakura, J. Chem. Phys. 107, 9482 (1997).

[4] B. Farizon, M. Farizon, H. Razafinjanahary, and H. Chermette, Phys. Rev. B 60, 3821 (1999).

[5] H. Chermette and I. V. Ymmud, Phys. Rev. B 63, 165427 (2001).

[6] M. Barbatti, G. Jalbert, and A. C. Nascimento, J. Chem. Phys. 113, 4230 (2000).

[7] M. Barbatti, G. Jalbert, and A. C. Nascimento, J. Chem. Phys. 114, 7066 (2001).

[8] R. Prosmiti, P. Villareal, and G. Delgado-Barrio, J. Phys. Chem. 107, 4768 (2003).

[9] I. A. Howard, F. E. Leys, N. H. March, C. Van Alsenoy, J. A. Alonso, and A. Rubio, in Proceedings of the SPIE on Nanotechnology, edited by R. Vajtai, X. Aymerich, L. B. Kish, and A. Rubio (SPIE, Bellingham, WA, 2003), Vol. 5118, p. 331.

[10] J. Zweiback, R.A. Smith, T.E. Cowan, G. Hays, K.B. Wharton,
V.P. Yanovsky, and T. Ditmire, Phys. Rev. Lett. 84, 2634 (2000).

[11] M. J. Frisch, G. W. Trucks, H. B. Schlegel, G. E. Scuseria, M. A. Robb, J. R. Cheeseman, V. G. Zakrzewski, J. A. Montgomery, Jr., R. E. Stratmann, J. C. Burant, S. Dapprich, J. M. Millam, A. D. Daniels, K. N. Kudin, M. C. Strain, O. Farkas, J. Tomasi, V. Barone, M. Cossi, R. Cammi, B. Mennucci, C. Pomelli, C. Adamo, S. Clifford, J. Ochterski, G. A. Petersson, P. Y. Ayala, Q. Cui, K. Morokuma, D. K. Malick, A. D. Rabuck, K. Raghavachari, J. B. Foresman, J. Cioslowski, J. V. Ortiz, A. G. Baboul, B. B. Stefanov, G. Liu, A. Liashenko, P. Piskorz, I. Komaromi, R. Gomperts, R. L. Martin, D. J. Fox, T. Keith, M. A. Al-Laham, C. Y. Peng, A. Nanayakkara, C. Gonzalez, M. Challacombe, P. M. W. Gill, B. Johnson, W. Chen, M. W. Wong, J. L. Andres, C. Gonzalez, M. HeadGordon, E. S. Replogle, and J. A. Pople, Gaussian 98, Revision A. 7 (Gaussian, Inc., Pittsburgh, PA, 1998).

[12] U. Naher, S. Bjornholm, S. Frauendorf, F. Garcias, and C. Guet, Phys. Rep. 285, 245 (1997).

[13] J. A. Alonso, M. Barranco, M. Garcias, P. G. Reinhard, and E. Suraud, Fission Dynamics of Atomic Clusters and Nuclei, edited by J. da Providencia, D. M. Brink, F. Karpechine, and F. B. Malik (World Scientific, Singapore, 2001), p. 163.

[14] E. Deumens, A. Diz, R. Longo, and Y. Öhrn, Rev. Mod. Phys. 66, 917 (1994). 